\title{
Influence of center gas supply device on gas-solid flow in COREX shaft furnace through DEM-CFD model
}

https://doi.org/10.1515/ijcre-2020-0012

Received January 18, 2020; accepted May 3, 2020; published online July 6, 2020

\begin{abstract}
In order to develop the central gas flow in COREX shaft furnace, a new installment of center gas supply device (CGD) is designed. In this work, a coupled DEM-CFD model was employed to study the influence of CGD on gas-solid flow in COREX shaft furnace. The particle descending velocity, particle segregation behaviour, void distribution and gas distribution were investigated. The results show that the CGD affects the particles descending velocity remarkably as the burden falling down to the slot. Particle segregation can be observed under the inverse ' $V$ ' burden profile, and the influence of CGD on the particle segregation is unobvious on the whole, which causes the result that the voidage is slightly changed. Although the effect of CGD on solid flow is not significant, the gas flow in shaft furnace has an obvious change. Compared with the condition without CGD, in the case with CGD, the gas velocity is improved significantly, especially in the middle zone of the furnace, which further promotes the center gas distribution. Meanwhile, the pressure drop in the furnace with the installation of CGD is increased partly.
\end{abstract}

Keywords: center gas distribution; COREX shaft furnace; DEM-CFD; gas-solid flow; pressure drop.

*Corresponding authors: Heng Zhou, State Key Laboratory of Advanced Metallurgy, School of Metallurgical and Ecological Engineering, University of Science and Technology Beijing, Beijing, 100083, China, E-mail: zhouheng@ustb.edu.cn; Binbin Du, Steel Subsidiary Company, WISDRI Engineering \& Research Incorporation Limited, Wuhan, 430223, Hubei, China, E-mail: dubinbin0816@163.com

Shuyu Wang, Mingyin Kou, Zhiyong Tang, Jiahui Yang and Shengli Wu: State Key Laboratory of Advanced Metallurgy, School of Metallurgical and Ecological Engineering, University of Science and Technology Beijing, Beijing, 100083, China

Dianyu E: International Research Institute for Minerals, Metallurgy and Materials, Jiangxi University of Science and Technology, Nanchang, 330013, Jiangxi, China

\section{Introduction}

Gas-solid counter-current reactor is a moving bed in which gas flow upward through the particle bed while the charged solid particles descend from the top and flow toward the outlet. It plays a critical role in ironmaking, coal gasification, chemical catalysis and other industries due to its advantages of large capacity and low investment cost (Kunii and Levenspiel 1991; Spallina et al. 2014; Trambouze 1990). In particular, the ironmaking devices, such as blast furnace and reduction shaft furnace are typical gas-solid counter-current reactor. The gas-solid flow in ironmaking reactors has a significant impact on the inner characteristics, such as pressure, temperature and species distributions. Understanding such complex flow in ironmaking reactor is crucial to achieve the stable operation and improve the operating efficiency.

Recently, with the desiring of high efficiency, low consumption, less environmental impact and so on, large scale are inevitable (Ueda et al. 2010; Zhu 2009). However, large-scaled ironmaking reactors face some issues and one typical problem is un-matched gas-solid distribution. The outstanding representative is COREX reduction shaft furnace. Actually, the COREX productivity has an evolution from 0.4 million tons per year to 1 million tons per year, reaching current level of 1.5 million tons per year. The mean diameter of COREX shaft furnace increases from $4.83 \mathrm{~m}$ in COREX-1000 type to $8.3 \mathrm{~m}$ in COREX-3000 type (Eberle, Siuka, and Bohm 2006). However, the low metallization rate, high fuel consumption and high pressure drop arise in C-3000 shaft furnace (Li 2008; Wang, Zhou, and Li 2012). All the problems relate to the insufficient center gas distribution. In order to overcome the technical problems, two parallel beams of areal gas distribution (AGD) has been proposed and tested in the shaft furnace of COREX-3000. In the tentative design, two beams are installed, and the reducing gas can be blasted into the shaft center via the triangle-shaped void, who is generated downstream of the beam due to solid flow (Zhou et al. 2015). Although the AGD technology is proven effective in improving reduction rate in plant (Lin, Song, and Xia 2013), it led to other issues such as 
chocking of gas slots (Zhou et al. 2018). For this reason, recently, the shaft furnace in COREX-3000 was further modified where the most inspiring device is Center Gas Supply Device (CGD). The gas-solid flow in COREX shaft furnace with CGD will be featured by the initial gas distribution and complex geometry.

Over the past decades, micro- and macro-scopic properties of gas-solid flow in blast furnace has been extensively investigated by combining discrete element method (DEM) with computational fluid dynamics (CFD) (Adema, Yang, and Boom 2010; Hou et al. 2017; Matsuhashi et al. 2012; Natsui et al. 2015; Ueda et al. 2015; Zhou et al. 2011). The successful application of DEM-CFD prompts a detailed simulation and analysis of gas-solid flow in COREX process by this approach. For example, Hou et al. developed a full scale CFD-DEM model to study the gas-solid flow inside a shaft furnace (Hou et al. 2014), and the effects of discharge rate and two common burden structures on the thermal behaviour of gas-solid flow in the shaft furnace were also investigated (Hou, Li, and $\mathrm{Yu}$ 2015). Bai et al. investigated the influence of bed conditions on gas flow in a shaft furnace by DEM-CFD modelling. They confirmed that bed shape has a significant influence on the gas flow (Bai et al. 2017). These studies provide useful information on the gas-solid flow in COREX shaft furnace, while the gas-solid flow in a shaft furnace with CGD has not been fully understood. Recently, the author developed a three-dimensional DEM model to investigate the solid flow in the shaft furnace with CGD (Zhou et al. 2018), and the effect of geometrical configuration of CGD on solid residence time was also studied (Zhou et al. 2019). Zhang et al. studied the influence of CGD structure on burden descending behavior in shaft furnace through a DEM model (Zhang, Zou, and Luo 2019). However, gas flow in shaft furnace with CGD was not considered in these works. Although the gas distribution and inner characteristics in shaft furnace with CGD were investigated through a CFD model (Zhang, Luo, and Zou 2019), the bed porosity distribution is not considered and the voidage is assumed as the same for the whole bed. Besides, the simulation of gasparticle flow in MIDREX shaft furnace also provided useful information for the studying about COREX shaft furnace (Ghadi, Valipour, and Biglari 2017; Valipour and Saboohi 2007). Thus, it is necessary to achieve a detailed exploration of the gas-solid flow in COREX shaft furnace with CGD considering the effect of particle segregation on bed voidage distribution.

In this work, the influences of CGD on gas-flow in COREX shaft furnace were investigated through DEM-CFD model. The comparison of particle velocity and segregation in the shaft furnace with and without CGD was investigated. Then the voidage distribution and gas flow in shaft furnace was further studied. The superiority and feasibility of shaft furnace with CGD can be proved through the contrast of two types findings of this work.

\section{Model description}

In this work, the gas-solid flow is composed of a discrete solid phase and a continuous gas phase. The heat and mass transform between the gas-solid phases were not considered. The solid phase is described by DEM, originally proposed by Cundall and Strack (Cundall and Strack 1979). According to the Newton's second law of motion, a particle can have two types of motion: translational and rotational. And during its descending process, the particle may be affected by the neighbor particles, or contact with wall and interact with the surrounding fluid. The governing equations for translational and rotational motions of particle $i$ can be written as

$$
\begin{gathered}
m_{i}\left(\mathrm{~d} \boldsymbol{v}_{i}\right) / \mathrm{d} t=\sum_{j=1}^{k_{i}}\left(\boldsymbol{F}_{\mathrm{cn}, i j}+\boldsymbol{F}_{\mathrm{dn}, i j}+\boldsymbol{F}_{\mathrm{ct}, i j}+\boldsymbol{F}_{\mathrm{d} t, i j}\right)+\boldsymbol{F}_{\mathrm{pf}, i}+m_{i} \boldsymbol{g} \\
\boldsymbol{I}_{i}\left(\mathrm{~d} \omega_{i}\right) / \mathrm{d} t=\sum_{j=1}^{k_{i}}\left(\boldsymbol{T}_{i j}+\boldsymbol{M}_{i j}\right)
\end{gathered}
$$

where, $m_{i}, I_{i}, \boldsymbol{v}_{i}, \boldsymbol{\omega}_{i}$ represent the mass, the rotational inertia, the translational velocity and the rotational velocity of particle $i$, respectively. $k_{i}$ denotes the particle numbers which are contacted with particle $i$. The forces involved are: the gravitational force $m_{i} \mathbf{g}$, the particle-fluid interaction force $\boldsymbol{F}_{\mathrm{pf}, \mathrm{i}}$, and the contact forces between particles, which include the normal contact forces $\boldsymbol{F}_{\mathrm{cn}, i j}$ and damping forces $\boldsymbol{F}_{\mathrm{dn}, i j}$, the tangential contact force $\boldsymbol{F}_{\mathrm{ct}, i j}$ and the normal damping forces $\boldsymbol{F}_{\mathrm{dt}, i j}$. In addition, the particle $i$ bears two kinds of torque: tangential torque $\boldsymbol{M}_{t, i j}$ and rolling friction torque $\boldsymbol{M}_{r, i j}$, generated from the tangential force and rolling friction, respectively. All the forces and torques used in this model are listed in Table 1.

A DEM-CFD model was developed to simulate the fluid flow. The continuous fluid flow modelled by CFD was at the computational cell level (Yang, Zhou, and Yu 2015; Zhou et al. 2008), and it is described by the continuity and NavierStokes equations, given by,

$$
\begin{gathered}
\partial \epsilon \rho / \partial t+\nabla \cdot(\rho \epsilon \boldsymbol{u})=0 \\
\partial(\epsilon \rho \boldsymbol{u}) / \partial t+\nabla \cdot(\rho \epsilon \boldsymbol{u u})=-\nabla p+\nabla \cdot(\mu \epsilon \nabla \boldsymbol{u})+\rho \epsilon \mathbf{g}-\boldsymbol{F}_{p f}
\end{gathered}
$$

where, $\rho, \varepsilon, \boldsymbol{u}, p$ and $\mu$ represent the density, void fraction, velocity vector, pressure and viscosity of gas phase, respectively. $\boldsymbol{F}_{\mathrm{pf}}$ is the volumetric particle-fluid interaction force in a computational cell, given by, 
(a)
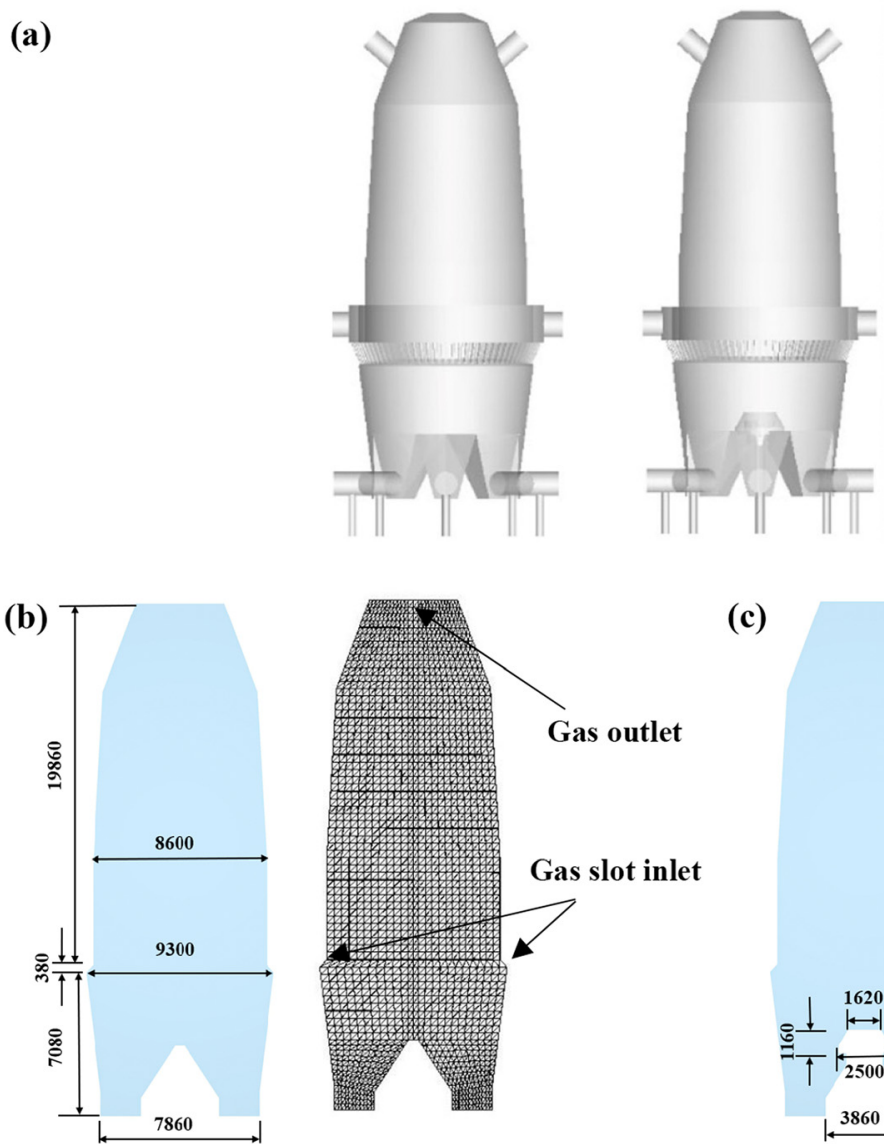

(c)

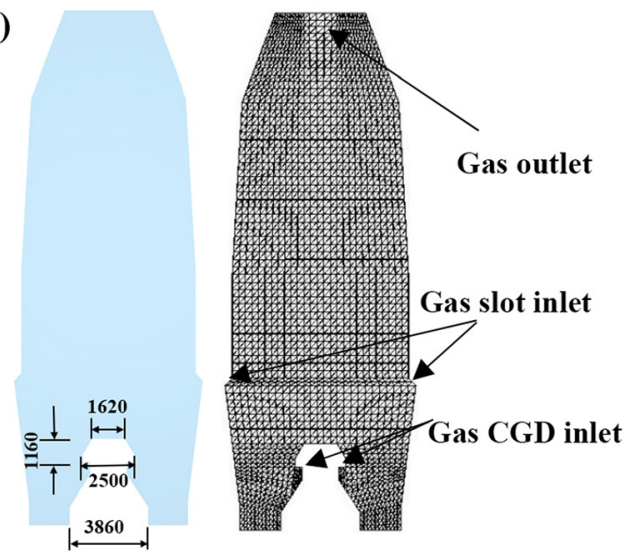

Figure 1: Schematic diagram of COREX shaft furnace (a), slot model and computational grid for the shaft without CGD (b), and with CGD (c) (Unit. mm).

\section{Results and discussion}

\subsection{Model validation}

In order to verity the feasibility of the DEM model, the simulation results are compared with the physical experimental measurements in the literature (Lee 1999). Among them, the material used in the physical experiment is magnetite. In the model, the shape of particles is spherical, and the physical properties of particles are corrected. The solid flow patterns of two conditions are shown in Figure 2. From the picture, it is clear that the stream-lines of solids came from simulation results is similar to the experimental results. That is to say, the particles are descending uniformly in the upper part of the shaft furnace, and the stream-lines are gradually changed to the " $\mathrm{W}$ " shape in the downward process; meanwhile, the calculated characteristic flow areas such as stagnant zone are basically consistent with the measured values of experiment. As a whole, the simulated burden descending behaviors are basically consistent with the physical experimental result, which confirms the feasibility and rationality of using the current model to predict the solid flow in the COREX shaft furnace.

\subsection{Particle velocity and segregation}

In this section, five samples are collected along the radius direction at different height for the exploration of particle descending and segregation behavior. For particle velocity, the particle average velocity in each sample can be obtained. And the total mass of each burden in each sample is collected in order to calculate the particles segregation. The detailed analyses are as follows.

Figure 3 shows the variation of velocity of particles with time in COREX shaft furnace with or without CGD while the particles reach a steady state. The variation tendency of both two furnace structure is similar, that is, the velocity of particles reaches the sharp peak value with the burden charging, which may have an impact on the moving particles, and thus cause the fluctuation of velocity; the narrow fluctuation range of velocity is observed in the 
Table 2: Material properties and simulation parameters.

\begin{tabular}{|c|c|c|c|c|c|c|}
\hline Parameters & & Pellet & Ore & Coke & Flux & Wal \\
\hline Density, $\rho\left(\mathrm{kg} / \mathrm{m}^{3}\right)$ & & 3425 & 4760 & 1100 & 2800 & 7850 \\
\hline Shear modulus, $G(\mathrm{MPa})$ & & 10 & 10 & 2.2 & 10 & 79,000 \\
\hline Poisson's ratio, $u(-)$ & & 0.25 & 0.21 & 0.22 & 0.21 & 0.30 \\
\hline Diameter, $d(\mathrm{~mm})$ & & 100 & 130 & 210 & 150 & - \\
\hline Mass ratio (wt\%) & & 51 & 34 & 7 & 8 & - \\
\hline Number of particles (-) & & 440,000 & 96,052 & 20,193 & 24,860 & - \\
\hline \multirow[t]{4}{*}{ Restitution coefficient, $e(-)$} & Pellet & 0.2 & 0.3 & 0.3 & 0.3 & 0.5 \\
\hline & Ore & - & 0.5 & 0.3 & 0.3 & 0.5 \\
\hline & Coke & - & - & 0.5 & 0.3 & 0.5 \\
\hline & Flux & - & - & - & 0.3 & 0.5 \\
\hline \multirow[t]{4}{*}{ Static friction, $\mu_{s}(-)$} & Pellet & 0.5 & 0.5 & 0.5 & 0.5 & 0.4 \\
\hline & Ore & - & 0.5 & 0.5 & 0.5 & 0.4 \\
\hline & Coke & - & - & 0.6 & 0.5 & 0.4 \\
\hline & Flux & - & - & - & 0.5 & 0.4 \\
\hline \multirow[t]{4}{*}{ Rolling friction, $\mu_{r}(-)$} & Pellet & 0.02 & 0.05 & 0.05 & 0.05 & 0.05 \\
\hline & Ore & - & 0.05 & 0.05 & 0.05 & 0.05 \\
\hline & Coke & - & - & 0.05 & 0.05 & 0.05 \\
\hline & Flux & - & - & - & 0.05 & 0.05 \\
\hline Burden discharging rate $(\mathrm{N} / \mathrm{s})$ & & & & & & 610 \\
\hline Air velocity without $C G D(\mathrm{~m} / \mathrm{s})$ & & & & & & 20 \\
\hline Air velocity with $\mathrm{CGD}(\mathrm{m} / \mathrm{s})$ & & & & & & $8 / 12$ \\
\hline Air density $\left(\mathrm{kg} / \mathrm{m}^{3}\right)$ & & & & & & 1.205 \\
\hline Air viscosity $(\mathrm{kg} / \mathrm{m} \mathrm{s})$ & & & & & & $1.8 \times 10^{-5}$ \\
\hline
\end{tabular}

(a)

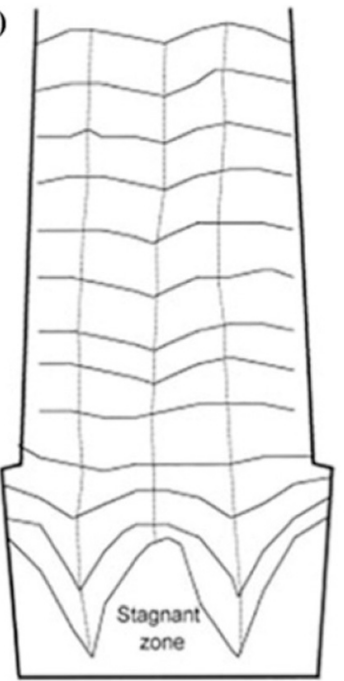

(b)

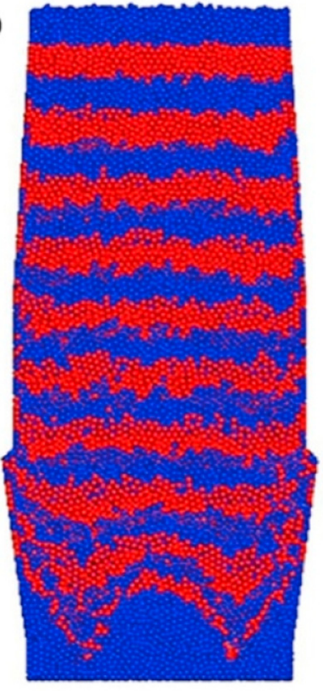

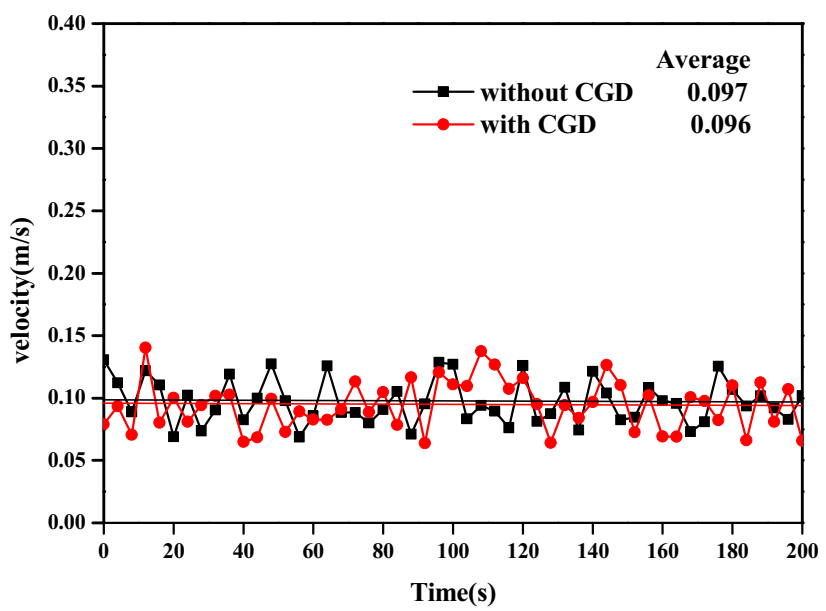

Figure 3: The velocity variation of particles in COREX shaft furnace (a) without or (b) with CGD.

Figure 2: Comparison of solid flow patterns between experimental measurement (a), and model prediction (b) in a slot model.

interval between the peaks; moreover, the magnitude of the average velocity for two cases is slight difference.

In order to have a further understanding of the influence of CGD on solid flow in COREX shaft furnace, three levels are used to represent the burden descending velocity, at the height of 15.0, 10.0 and $6.0 \mathrm{~m}$ above the bottom, respectively. The comparison of burden descending

velocity along the radius direction between cases with and without CGD is shown in Figure 4. The velocities investigated in this work are the average velocities of relatively stable period. From Figure 4(a), it is clear that the burden descending velocity at height $15.0 \mathrm{~m}$ along the radius direction is quite similar in both cases, and the particles velocity close to the wall is slower apparently than that of in furnace center due to the boundary effect. This phenomenon indicates that the installation of CGD in the shaft 


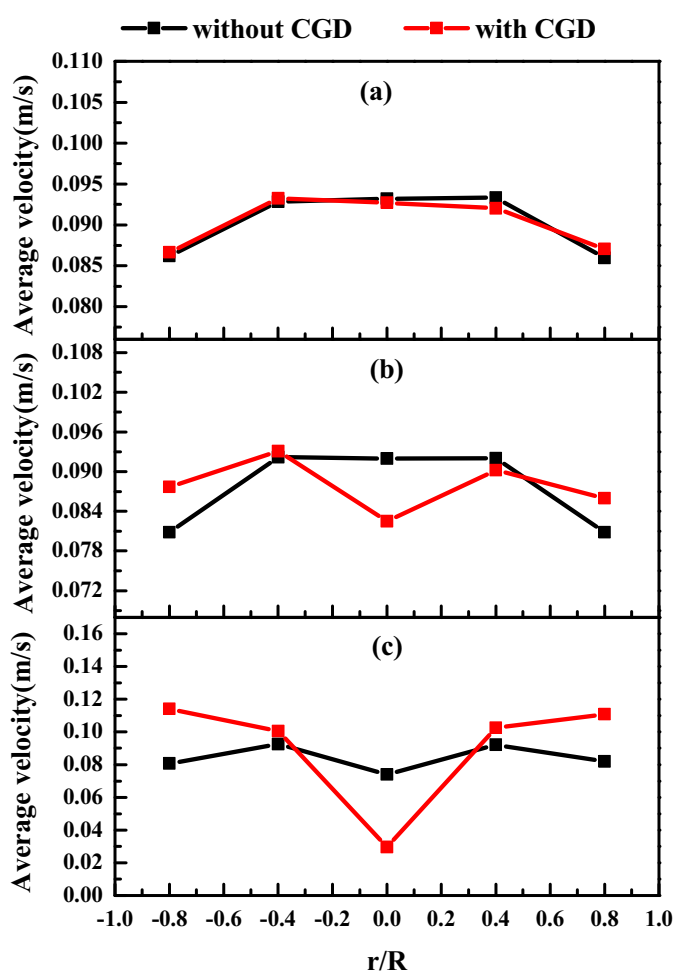

Figure 4: Burden descending velocity along the radius direction at different height above bottom in shaft furnace without or with CGD, (a) $15.0 \mathrm{~m}$; (b) $10.0 \mathrm{~m}$; (c) $6.0 \mathrm{~m}$.

furnace have little influence on burden descending velocity in the upper part of shaft furnace.

The burden descending velocity at height $10.0 \mathrm{~m}$ is shown in Figure 4(b). It can be seen that, the velocities of two cases reveal the difference. The velocity evolution of the case without CGD is quite similar with the results of the height $15.0 \mathrm{~m}$. However, it should pay attention to the case with CGD, due to the inhibition of CGD, the particles velocity in the center is quite smaller than that of the case without CGD, while the particles near the wall fall down faster. Although the velocity shows difference along the radius direction, the average velocities are similar. What's more, the descending velocity of particles located in the upper part of the screw is the highest in both two cases.

Figure 4(c) shows the burden descending velocity at height $6.0 \mathrm{~m}$, which is close to the top of CGD. The difference of velocities evolution between the cases with or without CGD is apparent. With the effect of guiding cone, the falling of particles in the furnace center was blocked, and thus the velocity is quite slow in both two cases; and the velocity in case with CGD is slower obviously because of the further influence of CGD. It is worth noting that in the case with CGD, the descending velocity close to the wall is the fastest along the radius direction, that may be due to

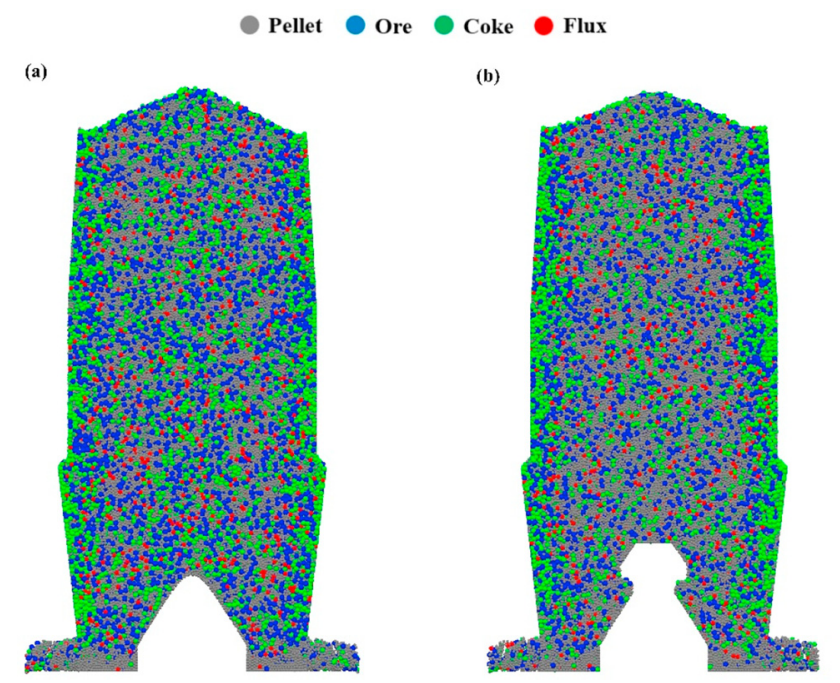

Figure 5: The distribution of particles in COREX shaft furnace (a) without or (b)with CGD.

the combined action of the burden profile and the mounting of the CGD.

Then the particle distribution in shaft furnace with or without CGD is explored and displayed in Figure 5. As the burden distribution changes slightly at stable condition, the instantaneous state selected randomly is used in this work. Different burdens filling in the shaft furnace are labeled with different colors, where pellet, ore, coke and flux particles are gray, blue, green and red color, respectively. As shown in Figure 5, inverse ' $V$ ' shape burden profile is generated in the upper of the furnace in both cases with or without CGD, and the particle segregation is obvious. The large particles such as coke and ore are on the edge of the furnace, while the center of shaft furnace is mainly filled with pellet. Moreover, this phenomenon is some more prominent in the case with CGD. In order to further quantitate the particle distribution, a particle segregation index is defined in the following study.

For particle segregation, the mass ratio of each burden in the above-mentioned sample can be calculation. Then, a segregation index is defined to assess the particle segregation, and the formula can be descripted as $(\mathrm{Ku}, \mathrm{Li}$, and Løvås 2013; You et al. 2019),

$$
N_{k}=\frac{P_{k}-P_{0, k}}{P_{0, k}}
$$

where, $P_{k}$ is the mass fraction of the burden materials in the sample, $P_{0, k}$ is the initial mass fraction of burden materials, and $k$ represents different burden. It is obvious that the $N_{k}$ reflects the segregation degree, that is, the larger absolute value of $N_{k}$ would bring out a more obvious particle segregation phenomenon. 
(a)
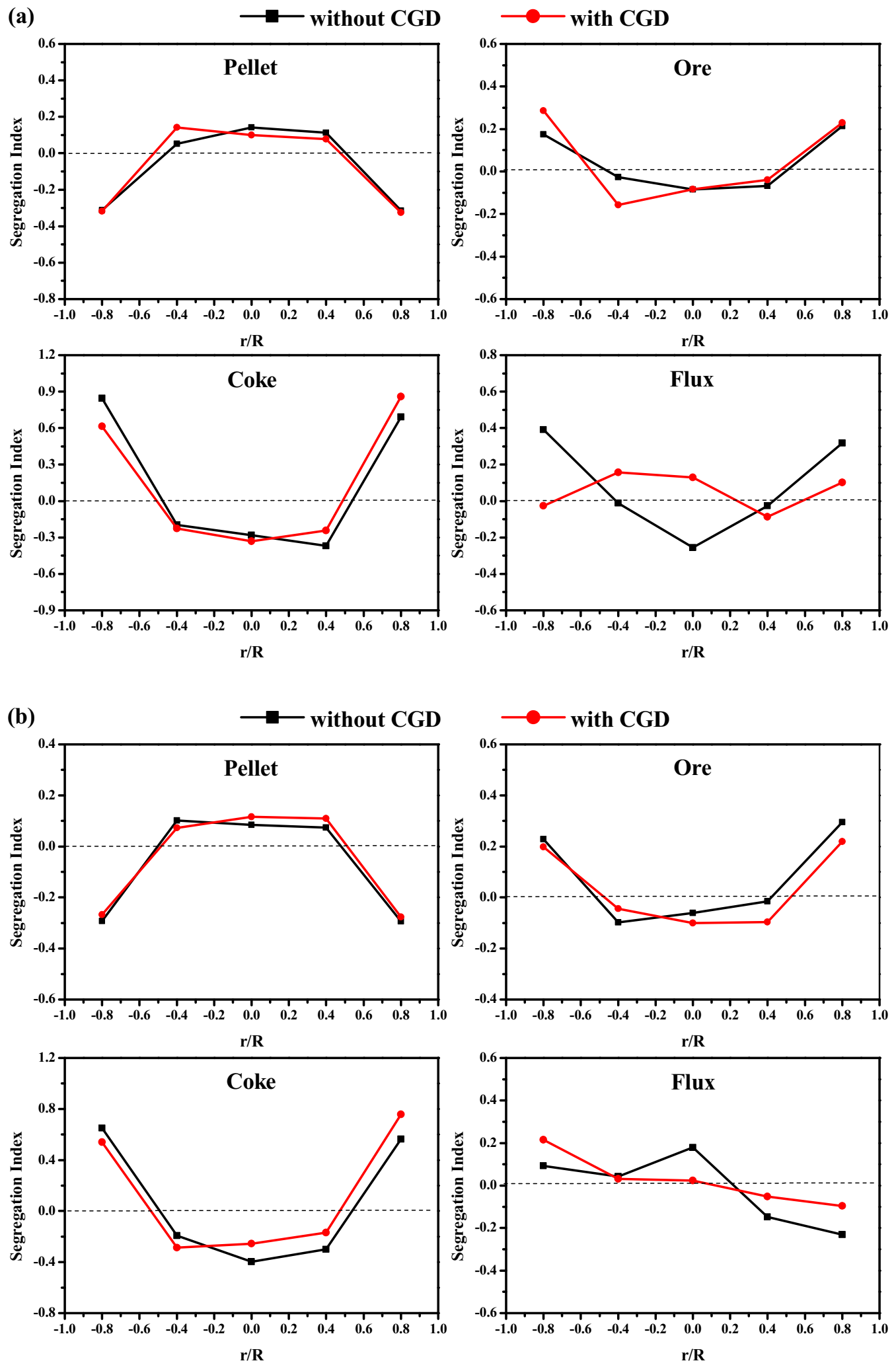

Figure 6: 6 Particle segregation along the radius direction at different height above bottom in shaft furnace with or without CGD, (a) 15.0m; (b) $6.0 \mathrm{~m}$. 


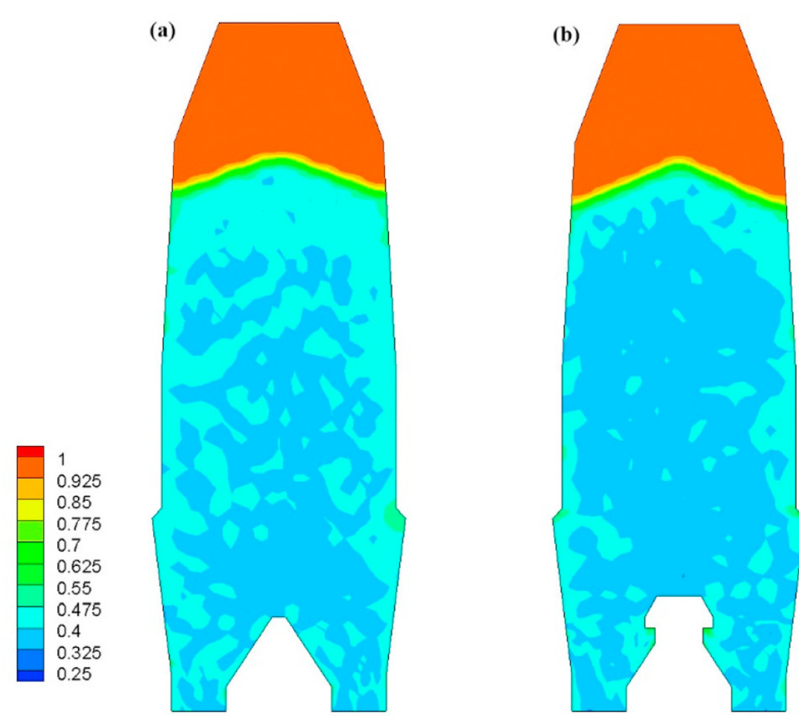

Figure 7: Void distribution in COREX shaft furnace (a) without or (b) with CGD.

Figure 6 shows the comparison of particle segregation along the radius direction between two cases at the height of 15.0 and $6.0 \mathrm{~m}$ above the bottom. As shown in Figure 6(a), in both two cases, with the landing point appear in the center, the pellet has a positive segregation in the center area while it presents negative near the furnace wall, and the tendency of coke and ore is on the contrary. This indicates that pellet is more likely to stay in the middle area, while the larger particles, such as coke and ore tend to roll from the landing point to the wall area. That is because the granular size of coke and ore is larger, particularly the coke has the smallest density among all the particles. The distribution of particle segregation at the height of $6.0 \mathrm{~m}$ is shown in Figure 6(b). Contrast to the result in Figure 6(a), the variation tendency of particle segregation along the radius direction is basically consistent. And the absolute value of the segregation index of coke is smaller slightly, that is to say the segregation phenomenon is more obviously in the upper of furnace.

As a whole, the influence of CGD on particles descending behaviors is inapparent, especially on the burden segregation, that illustrates the feasibility to install the CGD in the COREX shaft furnace.

\subsection{Voidage and gas distribution}

Figure 7 shows the void distribution in COREX shaft furnace with or without CGD. The void distribution is directly determined by the particle mass ratio and segregation. From Figure 7, it can be observed that the largest void is in the wall zone while the most pellet stay in the

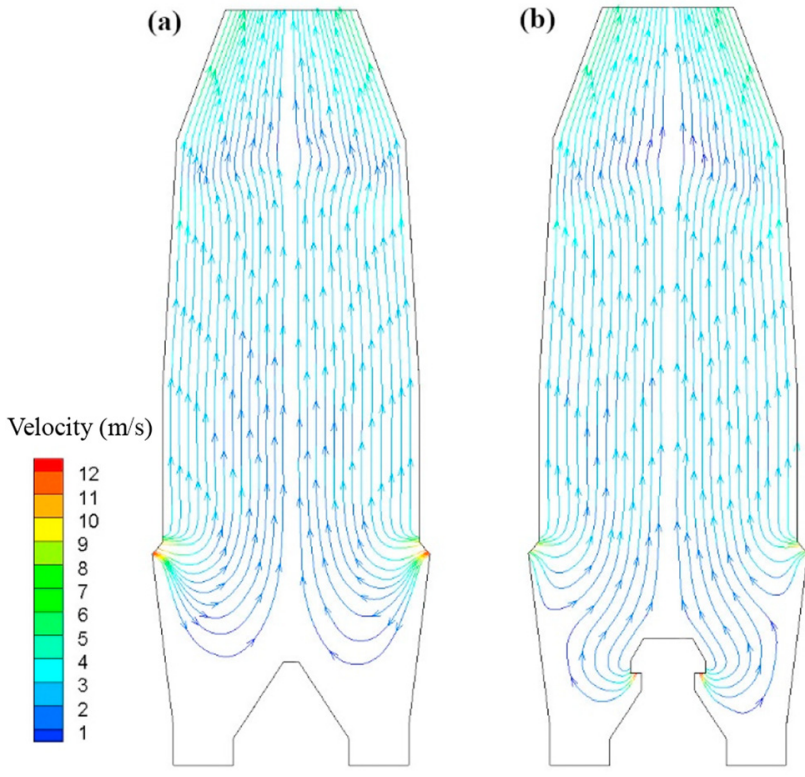

Figure 8: The streamline of the gas flow in COREX shaft furnace (a) without or (b) with CGD.

center area, and leading to the smaller void. And compared with the furnace without CGD, the overall voidage of shaft furnace with CGD is somewhat smaller, particularly in the middle zone.

To further study the effect of CGD on the gas flow, the gas was injected from the slot or CGD of the shaft furnace after the burden was steady. The streamline of the gas flow with or without CGD is shown in Figure 8. Compare Figure 8(a) with (b), the gas distribution injected from the slot of the furnace show a 'J' shape. And for the case with CGD, the gas was injected from both slot and CGD of the furnace, and that enriches the central gas distribution of the lower part of the shaft furnace.

The gas radial distribution at different height is discussed for the further investigation of gas flow in the shaft furnace. And Figure 9(a) shows the radial distribution of gas velocity at height $5.5 \mathrm{~m}$ that in the upside of the CGD. The gas distribution of two cases vary dramatically due to the influence of the installation of CGD. For the case with CGD, the gas velocity is increased initially and then decreased from center to wall, while the gas distribution of furnace without CGD vary significantly. It can be observed that the gas velocity of furnace with CGD in the middle zone (the absolute value from the center is in the range of 1 to $2.7 \mathrm{~m}$ ) is much larger than that of the case without CGD, while in the wall area, the case without CGD has the larger velocity. All that indicated that the influence of the CGD on the gas distribution below the slot of furnace is significant, it can enhance the gas flow in the center of the furnace and thus may improve the reduction rate of furnace. 

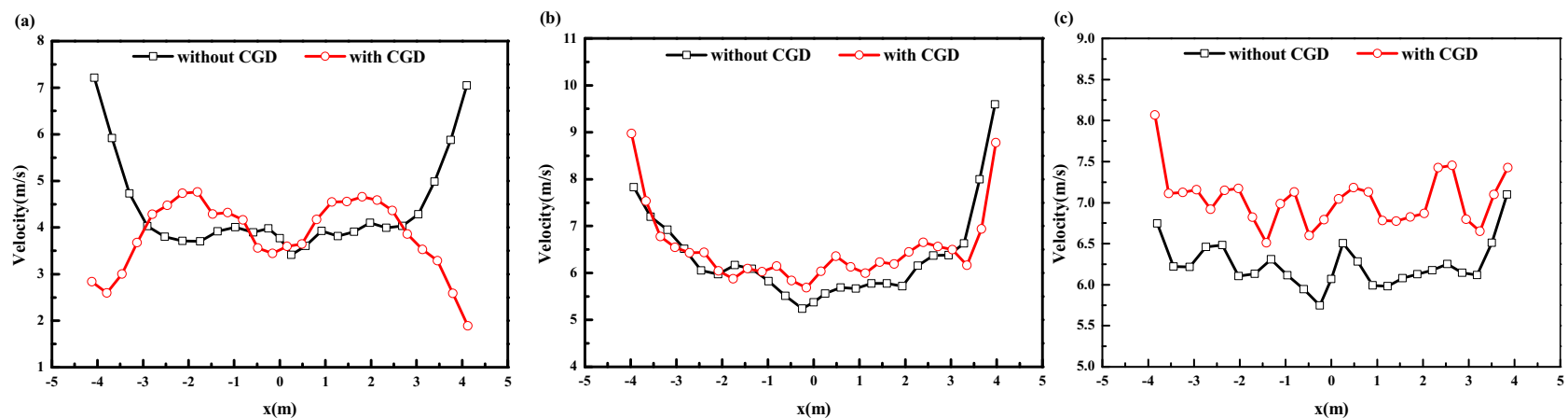

Figure 9: The radial distribution of gas velocity at height (a) $5.5 \mathrm{~m}$, (b) $8 \mathrm{~m}$, and (c) $15 \mathrm{~m}$.

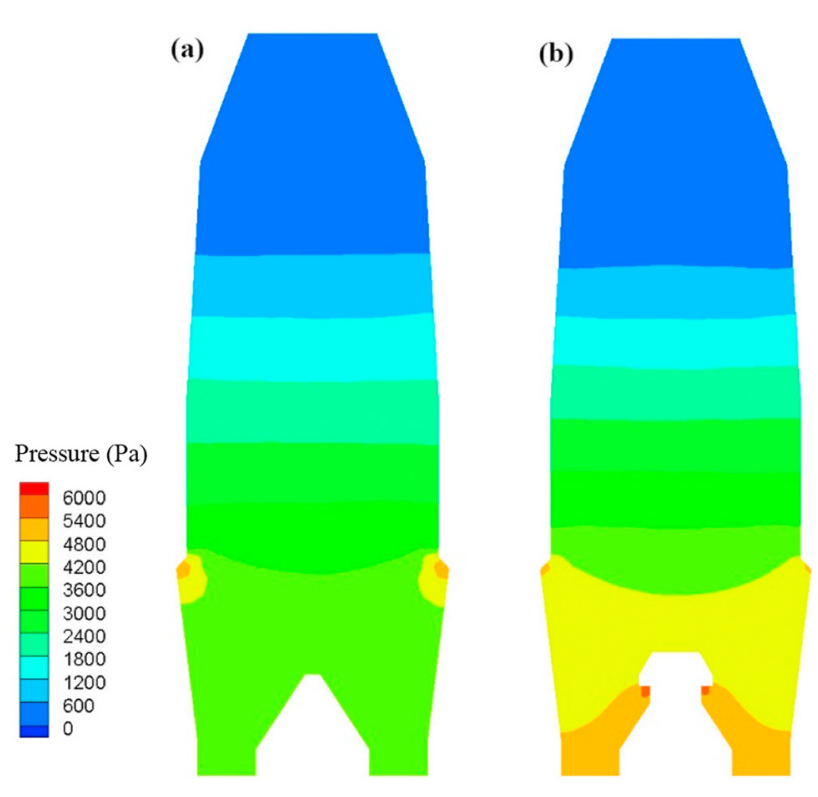

Figure 10: The pressure drop in COREX shaft furnace (a) without or (b) with CGD.

Figure 9(b) reveals the gas radial distribution at height $8 \mathrm{~m}$ (near the upper of the slot). For the condition with CGD, the velocity increases with the increase of height, for example, the gas velocity increases from 3.44 to $6.04 \mathrm{~m} / \mathrm{s}$ in the center area. The variation tendency of two cases is mainly consistent, that is, the velocity near the wall area is larger than that of in the center zone, but the interval between maximum and minimum of furnace with CGD is slightly narrower. The main reason is the "wall effect". As the void fraction near the walls are larger than that in the center zone, the gas velocity is higher near the walls. Moreover, the gas velocity in the middle area of that case is significantly higher while that of two cases is adjacent near the wall. As seen in Figure 9(c), the overall gas velocity increases when gas continues to move upwards. At height $15 \mathrm{~m}$, the velocity of furnace with CGD is obviously higher than that of the case without CGD. What's more, by the contrast between the wall and center gas velocity for both two cases, the maximum gas velocity near the wall are 19 and $12.6 \%$ higher than that in the center respectively, that is to say the gas distribution is more uniform and the central gas flow is more sufficient in the furnace with CGD.

As a whole, the overall gas velocity is improved and the center gas distribution is promoted apparently with the installation of CGD, and thus the metallization rate and gas utilization ratio may further be improved.

And the pressure drop in the shaft furnace with or without CGD is further explored to comprehend the effect of CGD on gas flow broadly. The isobar distribution of both two cases is shown in Figure 10. It is obvious that the isobar is basically horizontal for both two cases in the upper and
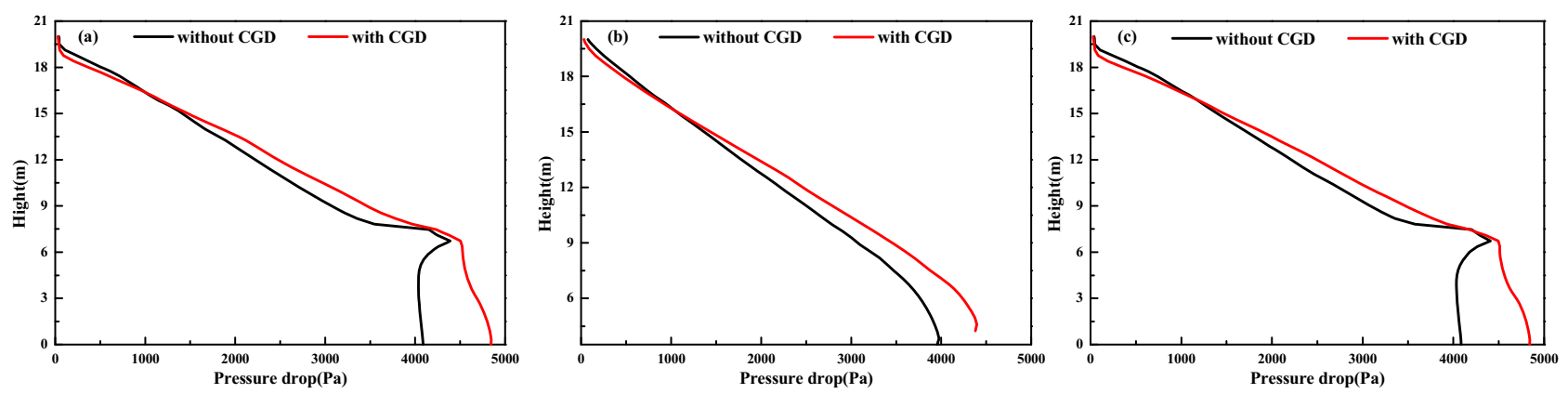

Figure 11: Longitudinal pressure drop at different position from center in shaft furnace. (a) $-3.8 \mathrm{~m}$; (b) $0 \mathrm{~m}$; (c) $3.8 \mathrm{~m}$. 
middle part of the furnace. And it presents ' $U$ ' shape near the slot inlet, the largest pressure drop is at the slot zone in the condition without CGD, while that is at the lower part of the furnace with CGD. What's more, it can be seen that the furnace with CGD has the larger pressure drop, and its largest pressure drop is $6696 \mathrm{~Pa}$ while that of the case without CGD is just 5939Pa. The longitudinal pressure drop at different position from center in shaft furnace is described in Figure 11. Among them, Figure 11(a) and (c) are the results on the left and right side of the symmetric position in the furnace, and Figure 11(b) depicts the longitudinal pressure drop at center position. On the one hand, it can be seen that the pressure drop in shaft furnace is extraordinary symmetric, which is consistent with the result of Figure 10. On the other hand, when the height exceeds $6 \mathrm{~m}$ (above slot of the furnace), the pressure drop increases linearly along the longitudinal direction for all the position, and the pressure drop reaches the maximum at the slot level for the position of $3.8 \mathrm{~m}$ from the center in the furnace without CGD, while the pressure drop continues to rise gently in another case. Furthermore, the pressure drop in the furnace with CGD is higher than that of the case without CGD consistently for all the position, this is because the gas injected from the CGD undergoes the higher packing bed when moving upward.

\section{Conclusions}

In this work, a coupled DEM-CFD model is developed to investigate the influence of CGD on gas-solid flow in COREX shaft furnace. And it principally pays attention on the exploration of particle velocity, particle segregation, void distribution, gas distribution and pressure drop. The mainly conclusions are summarized as following:

(1) The descending velocity of particles located in the upper of furnace is almost unaffected with the installation of CGD. However, the particles were hindered by the CGD with the descending process, especially the burden in the middle area of the furnace, resulting in the lower velocity compared to the case without CGD.

(2) Under the inverse ' $\mathrm{V}$ ' burden profile, particle segregation can be observed significantly. The large particles like coke and ore would like to roll to the wall zone, while the pellets tend to stay in the landing point. And contrast two cases, the influence of CGD on the particle segregation is indistinctive on the whole.

(3) The void distribution is affected by the CGD slightly. Meanwhile, the effect of CGD on the gas velocity is obvious, especially below the slot zone; the installment of CGD improves the gas velocity and promotes the center gas distribution. What's more, the CGD can increase the pressure drop in the furnace.

(4) Though the appliance of CGD increases the pressure drop partly, the center gas flow was developed significantly with the little influence on the solid flow behavior, which can further promote to obtain the higher metallization rate and gas utilization ratio.

Author contribution: All the authors have accepted responsibility for the entire content of this submitted manuscript and approved submission.

Research funding: The authors would like to thank the National Natural Science Foundation of China (Grant Number: 51904023, 51804027) and Fundamental Research Funds for the Central Universities (Grant Number: FRF-TP19-035A2) for their financial supports.

Conflict of interest statement: The authors declare no conflicts of interest regarding this article.

\section{References}

Adema, A. T., Y. X. Yang, and R. Boom. 2010. “Discrete Element Method-Computational Fluid Dynamic Simulation of the Materials Flow in an Iron-making Blast Furnace.” ISIJ International 50: 954-61.

Bai, M. H., S. F. Han, W. Y. Zhang, K. Xu, and H. Long. 2017. “Influence of Bed Conditions on Gas Flow in the COREX Shaft Furnace by DEM-CFD Modelling.” Ironmaking \& Steelmaking 44: 685-91.

Cundall, P. A., and O. D. L. Strack. 1979. “A Discrete Numerical Model for Granular Assemblies.” Geotechnique 29: 47-65.

Eberle, A., D. Siuka, and C. Bohm. 2006. "New Corex C-3000 Plant for Baosteel and Status of the Corex Technology." Stahl und Eisen 126: $31-40$.

Ghadi, A. Z., M. S. Valipour, and M. Biglari. 2017. "CFD Simulation of Two-phase Gas-particle Flow in the Midrex Shaft Furnace: The Effect of Twin Gas Injection System on the Performance of the Reactor.” International Journal of Hydrogen Energy 42: 103-18.

Hou, Q. F., M. Samman, J. Li, and A. B. Yu. 2014. “Modeling the Gassolid Flow in the Reduction Shaft of COREX." ISIJ International 54: 1772-80.

Hou, Q. F., J. Li, and A. B. Yu. 2015. “CFD-DEM Study of Heat Transfer in the Reduction Shaft of Corex." Steel Research International 86: 626-35.

Hou, Q. F., D. Y. E. S. B. Kuang, Z. Y. Li, and A. B. Yu. 2017. “DEM-based Virtual Experimental Blast Furnace: A Quasi-steady State Model.” Powder Technology 314: 557-66.

Kou, M. Y., J. Xu, H. Zhou, B. J. Wen, K. Gu, S. Yao, and S. L. Wu. 2018. "Effects of Bottom Base Shapes on Burden Profiles and Burden Size Distributions in the Upper Part of a COREX Shaft Furnace Based on DEM." Advanced Powder Technology 29: 1014-24.

Ku, X., T. Li, and T. Løvås. 2013. “Influence of Drag Force Correlations on Periodic Fluidization Behavior in Eulerian-Lagrangian 
Simulation of a Bubbling Fluidized Bed." Chemical Engineering Science 95: 94-106.

Kuang, S. B., M. M. Zhou, and A. B. Yu. 2020. "CFD-DEM Modelling and Simulation of Pneumatic Conveying: A Review." Powder Technology 365: 186-207.

Kunii, D., and O. Levenspiel. 1991. Fluidization Engineering. Boston: Butterworth Heinemann.

Lee, Y. J. 1999. "A Scaled Model Study on the Solid Flow in a Shaft Type Furnace.” Powder Technology 102: 194-201.

Li, W. G. 2008. Operation status Quo and Technical problems of COREX-3000, Baosteel Technol: 11-8.

Lin, J. J., W. G. Song, and W. Y. Xia. 2013. "Progress and Improving Directions of COREX-3000 in Baosteel." The 5th Baosteel Biennial Acad. Conf., SSTLPH, Shanghai, A499-506.

Matsuhashi, S., H. Kurosawa, S. Natsui, T. Kon, S. Ueda, R. Inoue, and T. Ariyama. 2012. "Evaluation of Coke Mixed Charging Based on Packed Bed Structure and Gas Permeability Changes in Blast Furnace by DEM-CFD Model." ISIJ International 52: 1990-9.

Natsui, S., H. Takai, R. Nashimoto, T. Kikuchi, and R.O. Suzuki. 2015. "Model Study of the Effect of Particles Structure on the Heat and Mass Transfer Through the Packed Bed in Ironmaking Blast Furnace." International Journal of Heat and Mass Transfer 91: $1176-86$

Spallina, V., M. C. Romano, P. Chiesa, F. Gallucci, M. van Sint Annaland, and G. Lozza. 2014. "Integration of Coal Gasification and Packed Bed CLC for High Efficiency and Near-zero Emission Power Generation.” International Journal of Greenhouse Gas Control 27: 28-41.

Trambouze, P. 1990. “Countercurrent Two-phase Flow Fixed Bed Catalytic Reactors." Chemical Engineering Science 45: 2269-75.

Ueda, S., S. Natsui, H. Nogami, J. Yagi, and T. Ariyama. 2010. "Recent Progress and Future Perspective on Mathematical Modeling of Blast Furnace." ISIJ International 50: 914-23.

Ueda, S., T. Kon, H. Kurosawa, S. Natsui, T. Ariyama, and H. Nogami. 2015. "Influence of Shape of Cohesive Zone on Gas Flow and Permeability in the Blast Furnace Analyzed by DEM-CFD Model." ISIJ International 55: 1232-6.

Valipour, M. S., and Y. Saboohi. 2007. "Numerical Investigation of Nonisothermal Reduction of Hematite Using Syngas: the Shaft Scale Study." Modelling and Simulation in Materials Science and Engineering 15: 487-507.

Wang, C., Z. Q. Zhou, and X. Q. Li. 2012. Analysis of operation and improvement approach of COREX-3000, World Iron Steel: 44-9.

Wu, S. L., J. Xu, S. D. Yang, Q. Zhou, and L. H. Zhang. 2010. "Basic Characteristics of the Shaft Furnace of COREX $尺$ Smelting Reduction Process Based on Iron Oxides Reduction Simulation." ISIJ International 50: 1032-39.

Xu, J., S. L. Wu, M. Y Kou, and K. P. Du. 2013. "Numerical Analysis of the Characteristics Inside Prereduction Shaft Furnace and Its
Operation Parameters Optimization by Using a Threedimensional Full Scale Mathematical Model." ISIJ International 53: $576-82$.

Yang, W. J., Z. Y. Zhou, D. Pinson, and A. B. Yu. 2014. "Periodic Boundary Condition for DEM Simulation of Particle Flow in Cylindrical Vessels." Industrial \& Engineering Chemistry Research 53: 8245-56.

Yang, W. J., Z. Y. Zhou, and A. B. Yu. 2015. "Discrete Particle Simulation of Solid Flow in a Three-dimensional Blast Furnace Sector Model." Chemical Engineering Journal 278: 339-52.

You, Y., Y. Y. Li, Z. G. Luo, H. F. Li, Z. S. Zou, and R. Y. Yang. 2019. "Investigating the Effect of Particle Shape on the Charging Process in Melter Gasifiers in COREX." Powder Technology 351: $305-13$

Zhang, X. S., Z. S. Zou, and Z. G. Luo. 2019. "Influence of CGD Structure on Burden Descending Behavior in COREX Shaft Furnace.” Metallurgical Research \& Technology 116: 304.

Zhang, X. S., Z. G. Luo, and Z. S. Zou. 2019. "Performance Simulation and Optimization of COREX Shaft Furnace with Central Gas Distribution.” Metallurgical Research \& Technology 116: 112.

Zhou, Z. Y., H. P. Zhu, A. B. Yu, B. Wright, and P. Zulli. 2008. "Discrete Particle Simulation of Gas-solid Flow in a Blast Furnace." Computers \& Chemical Engineering 32: 1760-72.

Zhou, Z. Y., H. P. Zhu, B. Wright, A. B. Yu, and P. Zulli. 2011. “Gas-Solid Flow in an Ironmaking Blast Furnace-II: Discrete Particle Simulation." Powder Technology 208: 72-85.

Zhou, H., Z. G. Luo, Z. S. Zou, T. Zhang, and Y. You. 2015. "Experimental Study on Burden Descending Behavior in COREX Shaft Furnace with AGD Beams." Steel Research International 86: 1073-81.

Zhou, H., S. L. Wu, M. Y. Kou, Y. You, Z. G. Luo, B. Tang, H. Cheng, X. Li, and Z. S. Zou. 2018. "Experimental Study and Numerical Simulation of Dust Accumulation in Bustle Pipe of COREX Shaft Furnace with Areal Gas Distribution Beams.” Ironmaking \& Steelmaking. https://doi.org/10.1080/03019233.2018. 1470361.

Zhou, H., S. L. Wu, M. Y. Kou, Z. G. Luo, W. He, Z. S. Zou, and Y. S. Shen. 2018. "Discrete Particle Simulation of Solid Flow in a Large-scale Reduction Shaft Furnace with Center Gas Supply Device.” ISIJ International 58: 422-30.

Zhou, H., Q. F. Hou, M. Y. Kou, S. Yao, and S. L. Wu. 2019. “Discrete Element Method Study of Solid Descending and Residence Properties in COREX Shaft Furnace with Center Gas Supply Device." Journal of Iron and Steel Research International 26: 669-78.

Zhu, H. P., Z. Y. Zhou, R. Y. Yang, and A. B. Yu. 2007. "Discrete Particle Simulation of Particulate Systems: Theoretical Developments." Chemical Engineering Science 62: 3378-96.

Zhu, M. Y. 2009. Modern Metallurgy. Beijing: Metallurgy Industry Press. 